\title{
Growing Ranks of Advanced Practice Clinicians - Implications for the Physician Workforce
}

\author{
David I. Auerbach, Ph.D., Douglas O. Staiger, Ph.D., and Peter I. Buerhaus, Ph.D., R.N.
}

Tensing hroughout the history of modern American medicine, physicians have made up the vast majority of professionals who diagnose, treat, and prescribe medication to patients. Although demand for medical services has increased markedly over the years (and is projected to grow more rapidly as the population ages), the physician supply has grown relatively slowly. Increased delegation of work, new technology, and streamlined care processes can help practices meet patient needs with fewer physicians, but still require an increasing number of health professionals. ${ }^{1}$

Physician supply is constrained in the short run by long training times and in the longer run by medical school capacity and the number of accredited residency positions. Despite a 16\% increase in graduate medical education (GME) slots in recent years, the Association of American Medical Colleges (AAMC) recently projected that the supply of physicians will increase by only $0.5 \%$ per year between 2016 and 2030 .

A growing share of health care services are being provided by advanced practice registered nurses (APRNs), particularly nurse practitioners (NPs), who make up the majority of APRNs, and by physician assistants (PAs). NPs and PAs provide care that can overlap with care provided by physicians (both in primary care and increasingly in other specialties), and the AAMC recognizes this overlap in its physician-demand forecasts. The number of NPs and PAs is growing rapidly, in part because of shorter training times for such providers as compared with physicians and fewer institutional constraints on expanding educational capacity. Residencies aren't required for APRNs - though organizations are increasingly offering them - and education programs have proliferated: according to the American Association of Colleges of Nursing, the number of NP degree programs (master's or doctorate) grew from 282 to 424 between 2000 and 2016. Baccalaureate-prepared RNs typically require 2 to 3 years of graduate education to become certified NPs. PA programs typically take 2 years and also don't require residencies. According to the National Center for Education Statistics, the number of PA degree programs grew from 135 to 238 between 2000 and 2016.

These dynamics will have lasting effects on the composition of the health care workforce and on working relationships among health professionals. To take a closer look at these trends, we estimated the number of full-timeequivalent physicians, NPs, and PAs between 2001 and 2016 using data from the U.S. Census Bureau's American Community Survey, which included a roughly $0.4 \%$ sample of the U.S. population between 2001 and 2004 and a 1\% sample between 2005 and 2016. Because the Census didn't identify NPs until 2010, we obtained data on NPs from the National Sample Survey of Registered Nurses from 2000, 2004, and 2008. Figures were validated using data from health professional associations. The final data set includes 12,887 NPs, 12,801 PAs, and 166,103 physicians.

These data were used to project the number of NPs, PAs, and physicians through 2030 using methods described in greater detail elsewhere. ${ }^{2}$ Briefly, our model estimates the number of providers of various ages in each year as a function of both workforceparticipation patterns associated with age and estimates of differences among birth cohorts in rates of entry into each profession, which reflect institutional constraints. Our projections assume that age-related workforce-participation patterns will remain stable after 2016 and that the size of the workforce for birth cohorts that have not yet entered the labor force will resemble that of the five most recent cohorts. In the case of physicians, to better capture the expansion in medical education and throughput in recent years, we assume that the size of future cohorts will resemble the size of only the most recent (largest) cohort. In our prior work, this model has successfully forecast health care workforce trends. ${ }^{2}$

As shown in the table, between 2001 and 2010, workforce supply increased by roughly 150,000 physicians (an increase of $2.2 \%$ per year), 27,000 NPs (an increase of $3.9 \%$ ), and 44,000 PAs (an increase of 7.9\%). Between 2010 and 2016, the combined increase in NPs and PAs $(79,000)$ outpaced the increase in physicians $(58,000)$, although 


\begin{tabular}{|c|c|c|c|c|c|c|c|}
\hline \multirow[t]{2}{*}{ Provider Group } & \multicolumn{4}{|c|}{ No. of Full-Time Equivalents } & \multicolumn{3}{|c|}{ Average Annual Growth (\%) } \\
\hline & 2001 & 2010 & 2016 & $\begin{array}{c}2030 \\
\text { (projected) }\end{array}$ & $2001-2010$ & 2010-2016 & $\begin{array}{l}2016-2030 \\
\text { (projected) }\end{array}$ \\
\hline Physicians & 711,357 & 862,698 & 920,397 & $1,076,360$ & 2.2 & 1.1 & 1.1 \\
\hline Nurse practitioners & 64,800 & 91,697 & 157,025 & 396,546 & 3.9 & 9.4 & 6.8 \\
\hline Physician assistants & 44,282 & 88,047 & 102,084 & 183,991 & 7.9 & 2.5 & 4.3 \\
\hline
\end{tabular}

* Based on data from the American Community Survey (ACS) and the National Sample Survey of Registered Nurses. Estimates for NPs in 2001 are interpolated on the basis of data from the 2000 and 2004 surveys. Full-time equivalents are defined on the basis of reported usual weekly hours worked and a 40-hour workweek for NPs and PAs and a 50-hour workweek for physicians. NPs include a small number of certified nurse midwives who were not separately identified in the ACS because of their small numbers. PAs in the ACS reporting an associate's degree or less education were excluded. All estimates are based on sample weights provided in each survey.

the NP and PA workforces were roughly one tenth the size of the physician workforce in 2010. During this period, growth in the NP supply accelerated to nearly $10 \%$ per year, whereas growth in the PA supply slowed to $2.5 \%$ and growth in physician supply slowed to $1.1 \%$. The number of NPs and PAs per 100 physicians nearly doubled between 2001 and 2016, from 15.3 to 28.2 .

We project that these trends will continue through 2030. The number of full-time-equivalent physicians is expected to continue growing by slightly more than $1 \%$ annually, as increased retirement rates are offset by increased entry, whereas the numbers of NPs and PAs will grow by $6.8 \%$ and $4.3 \%$ annually, respectively. Roughly two thirds $(67.3 \%)$ of practitioners added between 2016 and 2030 will therefore be NPs or PAs, and the combined number of NPs and PAs per 100 physicians will nearly double again to 53.9 by 2030. These shifts will probably be even more pronounced in primary care, where physician supply has been growing more slowly than in other fields and NPs tend to be more concentrated.
The changing composition of the workforce will have implications for provider teams. Primary care providers, in particular, increasingly work in larger groups of professionals with varying backgrounds and types of training. A 2012 national survey of primary care NPs and physicians found that 8 in 10 NPs worked in collaborative practice arrangements with physicians and $41 \%$ of physicians worked with NPs - a percentage that will probably grow over time. ${ }^{3}$ As more states expand practice authority for NPs, medical practices will have to adjust. A recent study of working relationships between NPs and physicians on primary care teams in New York and Massachusetts found that physicians, other staff, and patients often confused the roles and skills of various providers and that these misunderstandings often led to practices undermining the productivity and efficiency of NPs. ${ }^{4}$ Physicians, NPs, and PAs will all need to be trained and prepared for this new reality.

Greater reliance on nonphysician clinicians is unlikely to threaten quality of care or increase costs. There is growing evidence that the primary care provided by NPs and PAs is similar to that provided by physicians, and a recent national study of Medicare beneficiaries found that the cost of primary care provided by NPs was significantly lower than the cost of physician-provided care. ${ }^{5}$

As with other projections, our findings are subject to some degree of uncertainty. It is unlikely that the physician supply will grow more rapidly than we project: the AAMC projects even slower growth, the number of GME slots is constrained, and even an immediate expansion of medical school capacity and training opportunities wouldn't substantially affect the physician supply for many years. Growth in the NP and PA workforces is more uncertain. Although shorter, more flexible training requirements for these providers have facilitated an unprecedented increase in new entrants, growth rates could fall if demand for nonphysician providers is lower than anticipated and job-market prospects worsen. Major changes are unlikely, however, given the expected increases in demand for care, growing use of team-based and interprofessional practice, and the fact that 
NPs disproportionately serve rural and underserved populations, whose needs would otherwise go unmet.

Despite these uncertainties, it is clear that patients will continue to encounter more NPs and PAs when they seek care. The shifting composition of the health care workforce will present both challenges and opportunities for medical practices as they redesign care pathways to accommodate new payment methods, new incentives regarding quality of care, and the demands of an aging population.

Disclosure forms provided by the authors are available at NEJM.org.

From the Center for Interdisciplinary Health Workforce Studies, College of Nursing, Montana State University, Bozeman (D.I.A., P.I.B.); the Department of Economics, Dartmouth College, Hanover, NH (D.O.S.); and the National Bureau of Economic Research, Cambridge, MA (D.O.S.).

1. Bodenheimer TS, Smith MD. Primary care: proposed solutions to the physician shortage without training more physicians. Health Aff (Millwood) 2013;32:1881-6.

2. Staiger DO, Auerbach DI, Buerhaus PI.
Comparison of physician workforce estimates and supply projections. JAMA 2009; 302:1674-80.

3. Donelan K, DesRoches CM, Dittus RS, Buerhaus P. Perspectives of physicians and nurse practitioners on primary care practice. N Engl J Med 2013;368:1898-906.

4. Poghosyan L, Norful AA, Martsolf GR. Primary care nurse practitioner practice characteristics: barriers and opportunities for interprofessional teamwork. J Ambul Care Manage 2017;40:77-86.

5. Perloff J, DesRoches CM, Buerhaus P. Comparing the cost of care provided to Medicare beneficiaries assigned to primary care nurse practitioners and physicians. Health Serv Res 2016;51:1407-23.

DOI: 10.1056/NEJMp1801869

Coppright @ 2018 Massachusetts Medical Society.

\title{
The Graduate Nurse Education Demonstration - Implications for Medicare Policy
}

\author{
Linda H. Aiken, Ph.D., R.N., Joshua Dahlerbruch, B.S.N., Barbara Todd, D.N.P., and Ge Bai, Ph.D., C.P.A.
}

$\mathrm{D}^{\mathrm{s}}$ espite decades of public and private investment, the United States continues to have a shortage of primary care capacity. Only 2699 graduating U.S. medical students - about $17 \%$ of graduates from allopathic and osteopathic schools - matched with primary care residencies in 2016. ${ }^{1}$ Studies show that nurse practitioners (NPs) provide highquality primary care that is satisfactory to patients, improves access to care in underserved areas, and may reduce costs of care. But although Medicare spends more than $\$ 15$ billion annually on graduate medical education $(\mathrm{GME}){ }^{2}$ including training for primary care physicians, it spends very little on clinical training for NPs.

Medicare has contributed to the cost of training nurses since its inception, but NP programs didn't exist when Medicare was enacted and such funding streams were established. Modernizing Medicare's payment policies for nurse training is highly relevant, given the recent success of the Graduate Nurse Education (GNE) Demonstration. ${ }^{3}$ The $\$ 200$ million, five-site Centers for Medicare and Medicaid Services (CMS) demonstration authorized under the Affordable Care Act showed that offering payments to Medicare providers enabled more of them to participate in clinical precepting of advanced practice registered nurses (APRNs) and resulted in a substantial increase in the number of new APRN graduates. More than $60 \%$ of training took place in community-based settings, and primary care NPs accounted for most of the growth in the number of new graduates.

The GNE Demonstration documented the success of a new model of organizing and paying for graduate nurse education involving consortia of hospitals and health systems, community partners, and university nursing schools managed by a single Medicare hospital hub. Such consortia were originally proposed in 1997 by the Institute of Medicine (now the National Academy of Medicine) as a strategy for increasing community-based training for physicians, but were not implemented until the GNE Demonstration. Of the five demonstration networks, three were state or regional consortia covering greater Philadelphia, the Texas Gulf Coast, and Arizona. In greater Philadelphia - the largest consortium - the Hospital of the University of Pennsylvania served as the designated hub for a regional network that included all health systems and hospitals in the area, more than 600 community-based providers, and all 9 local university nursing schools involved in training APRNs. This model has many advantages. For 